\title{
O ensino do spin: uma abordagem integrada a tecnologia e a sociedade
}

The teaching of spin: an approach integrated to tecnology and society

$$
\text { Marcos Paulo Belançon*1] }
$$

${ }^{1}$ Universidade Tecnológica Federal do Paraná, Departamento Acadêmico de Física, Grupo de Física de Materiais, Pato Branco, PR, Brasil

Recebido em 07 de Dezembro, 2017. Revisado em 20 de Março, 2018. Aceito em 07 de Abril, 2018.

\begin{abstract}
Neste trabalho apresento uma abordagem revisada do Spin para um curso introdutório de física moderna. Essa proposta é elaborada considerando o ponto de vista de que habitualmente o Spin é apresentado como um "objeto estranho"; ele sugere aos alunos que os elétrons estão girando basicamente no mesmo instante em que os alunos são apresentados a mecânica ondulatória, onde partículas clássicas não existem mais. Aqui, busco apresentar o Spin respeitando a ordem cronológica de sua concepção, discutindo os artigos originais e o que pensaram os principais autores que coletivamente contribuiram para a teoria. Paralelamente, isso é feito contextualizando a importância tecnológica que o Spin desempenha em nossa sociedade.
\end{abstract}

Palavras-chave: Spin, Tecnologia, Sociedade.

In this work I present an revised approach to the Spin for an introductory course of modern physics. This proposal is elaborated considering the point of view that oftenly the Spin is presented as a "strange object"; it suggests to the students that electrons are spinning basically in the same instant that those students are presented to ondulatory mechanics, where classical particles no longer exists. Here, I look for present the Spin regarding the cronological order as it was conceived, discussing the original articles and what though the main authors that collectivelly contributed to the theory. At the same time, this is made contextualizing the technological importance that is played by the Spin in our society.

Keywords: Spin, technology, society.

\section{Revisitando o Spin}

Como discutido num trabalho anterior [1], a reformulação do ensino de física para este século precisa ser feita considerando uma abordagem que intrinsicamente inclua os desdobramentos da ciência na tecnologia, política e sociedade. Essa necessária discussão sobre o ensino de física já é antiga, mas nossos livros continuam os mesmos, e eles são a pedra fundamental na imensa maioria dos cursos. Nós professores temos uma forte tendência a lecionar uma disciplina da maneira que ela nos foi lecionada, utilizando agora como professores o mesmo livro que utilizamos quando alunos.

Neste trabalho, compartilho uma revisão das contribuições de autores que foram fundamentais para a construção da teoria do Spin, revisando o tema e buscando contextualizar a sua importância a questões tecnológicas que permeiam nossa sociedade neste século. Esta revisão tem sido utilizada num curso introdutório de física moderna que leciono há alguns semestres.

Usualmente nossos sistemas de ensino nos apresentam o Spin do elétron durante um curso de química no ensino médio, quando estudamos os modelos atômicos e

*Endereço de correspondência: marcosbelancon@utfpr.edu.br então a distribuição eletrônica [2. Já nas universidades praticamente todos estudantes de exatas retomam o assunto com mais profundidade [2], entretanto, o ensino deste tópico permanece um desafio bastante particular. Os conceitos mais abstratos da mecânica clássica como o de momento ângular são atribuidos a uma partícula que, ao se conhecer a proposta de deBroglie e a subsequente formulação da teoria de Schrödinger, não pode mais ser tratada como uma "pequena bola de bilhar".

A apresentação do tema que faço a partir da seção a seguir se dá logo depois das aulas sobre a equação de Schrödinger, quando portanto os alunos já foram introduzidos aos conceitos de quantização, modelo atômico de Bohr para o átomo de hidrogênio e ondas de matéria de deBroglie.

\section{Introdução ao conceito de Spin}

Em 1897, portanto antes mesmo do nascimento da mecânica quântica, Pieter Zeeman documentou [3] o que ficou conhecido como efeito Zeeman. No trabalho publicado na Nature, já no primeiro parágrafo ele escreveu: “...me ocorreu que a frequêcia da luz emitida por uma chama poderia ser alterada se a chama estiver submetida a um campo 
magnético. Verifiquei que esta ação realmente ocorre". O efeito Zeeman chamou a atenção de muita gente, mas se tratava de uma alteração pequena nos espectros emitidos: basicamente cada linha espectral era dividida em duas outras, muito próximas da linha original.

É curioso notar que Zeeman ainda não havia se dado conta da existência do elétron, que estava sendo descoberto por Thomsom praticamente ao mesmo tempo que Zeeman fez seu trabalho de 1897. Inclusive, em outro parágrafo do artigo, Zeeman cita o Prof. Lorentz e sua teoria: “...nesta teoria é considerado que, em todos os corpos, existem pequenos elementos moleculares carregados com eletricidade, e que todos os processos elétricos são uma consequência do movimento ou do equílibrio de tais ions".

É conhecido que num átomo o elétron em torno do núcleo resulta num momento magnético. Este momento é previsto classicamente a medida que temos uma carga em torno do nucleo e isso é semelhante a uma espira de corrente; que portanto é sensível a campos magnéticos como um pequeno imã. Zeeman estava indiretamente medindo um outro momento magnético, que tem uma origem diferente desta do movimento eletrônico. Entretanto, sem ter um modelo atômico ou conhecer o elétron propriamente, com base na teoria de Lorentz ele imaginou que o efeito era devido a "rotação de tais ions". Hoje já sabemos que a afirmação da rotação do elétron não faz muito sentido, pois ele não é uma partícula no sentido clássico.

A questão da origem do momento magnético dos átomos perdurou por muito tempo [4], mas enfim se descobriu que, ao supor que os fenômenos magnéticos teriam origem na órbita do elétron, não deveriam existir imãs como conhecemos; eles seriam muito mais fracos. Além da espectroscopia, como no efeito Zeeman, havia então um índicio no magnetismo para se supor que o elétron possuiria um momento magnético próprio, além do seu momento magnético orbital.

Em 1926 o elétron e o próton já eram conhecidos e faltava apenas a descoberta do nêutron para se completar o modelo atômico. Naquele ano George Uhlenbeck e Samuel Goudsmit publicaram um trabalho entitulado [5] "elétrons girantes e a estrutura dos espectros" 1 . No artigo eles apresentam um novo modelo para explicar o fenômeno, no qual se postula a existência de momento magnético intrínseco; do mesmo modo que o elétron tem carga elétrica, ele possuiria intrinsecamente um momento magnético.

Schrödinger [6], por sua vez, cita nos parágrafos finais de seu artigo o que ele chama de "componentes espectrais de Zeeman", já sugerindo que uma formulação mais completa da mecânica ondulatória deveria incluir a relatividade restrita e o magnetismo, o que deveria de alguma forma explicar a origem do efeito Zeeman. Hoje sabemos que a hipótese de ambos estava correta, e a ideia de que

${ }^{1} \mathrm{O}$ trabalho foi submetido no ano anterior e publicado em Janeiro de 1926, enquanto o trabalho de Schrödinger foi publicado em dezembro do mesmo ano 6. o elétron possui momento magnético intrínseco explica de uma só vez a origem dos fenômenos magnéticos e a duplicidade das linhas de emissão do efeito Zeeman.

A existência dessa propriedade do elétron aumenta o número de níveis de energia que ele pode ocupar no átomo; para cada nível previsto pela equação de Schrödinger existem na verdade dois subniveis, em cada um o elétron possui spin para cima ou para baixo. Isto é conhecido como o príncipio de exclusão de Pauli: duas partículas não podem ocupar o mesmo estado quântico, i.e. possuir ao mesmo tempo a mesma função de onda. Se não bastasse isso, Pauling [7] mostrou um modelo para explicar as ligações químicas, no qual dois átomos compartilham os elétrons de valência de uma maneira só possível devido a existência do Spin. As peças do quebra-cabeça estavam se encaixando. Então, além dos três números quânticos que obtemos com a equação de Schrödinger existe um quarto número quântico: $o$ momento ângular intrinseco ou spin 2 .

\subsection{Os números quânticos e a distribuição eletrônica}

O número quântico principal " $n$ " define qual é a energia de um elétron naquele estado quântico; vários elétrons podem estar no mesmo nível energético, entretanto nunca no mesmo estado quântico. Isso quer dizer que se dois elétrons possuem o mesmo $n$, necessariamente precisarão ter valores diferentes para o número quântico de momento ângular orbital " $l$ ", azimutal " $m_{l}$ " ou de spin " $m_{s}$ ".

A primeira solução da equação de Schrödinger comportaria apenas um elétron; com $n=1$ não é possível que os outros dois números quânticos assumam valores diferentes de zero, logo, só há um estado quântico possível. No entanto, adicionando o spin o nível energético $n=1$ passa a comportar dois elétrons: o spin dos dois fica alinhado antiparalelamente, cada um em um estado quântico diferente. Nesta situação o momento magnético total é nulo. Nas nossas primeiras aulas de química somos apresentados a tabela periódica, vendo que na distribuição eletrônica devemos considerar os quatro números quânticos. O que nós não sabiamos lá no ensino médio quando fomos apresentados a essa distribuição, aparentemente sem sentido, é que ela resulta da solução da equação de Schrödinger. A tabela 1 apresenta alguns exemplos de orbitais eletrônicos e seus respectivos números quânticos.

Note que convencionamos $s \rightarrow l=0, p \rightarrow l=1$, $d \rightarrow l=2$ e $f \rightarrow l=3$; o expoente no orbital dá o número de elétrons no orbital. No Brasil é mais popularmente conhecido como diagrama de Pauling o esquema que explica em que ordem os elétrons vão se "alojando" no átomo, i.e. quando um átomo possui mais elétrons, qual nível é ocupado primeiro. É relativamente fácil imaginar que os níveis ocupados em primeiro lugar são aqueles com menor energia, portanto mais estáveis. Entretanto, não é

${ }^{2}$ Dirac 8$]$ fez uma formulação da mecânica quântica análoga a de Schrödinger que já contém todos os quatro números quânticos. A equação de Dirac é portanto mais completa que a de Schrödinger. 
Tabela 1: Números quânticos e os orbitais

\begin{tabular}{cccccc}
\hline $\mathbf{n}$ & $\mathbf{l}$ & $m_{l}$ & $m_{s}$ & $N^{o}$ de Elétrons & Orbital \\
\hline 1 & 0 & $(0)$ & $(\uparrow \downarrow)$ & 2 & $1 s^{2}$ \\
2 & 0 & $(0)$ & $(\uparrow \downarrow)$ & 2 & $2 s^{2}$ \\
2 & 1 & $(-1,0,1)$ & $(\uparrow \downarrow, \uparrow \downarrow, \uparrow \downarrow)$ & 6 & $2 p^{6}$ \\
3 & 0 & $(0)$ & $(\uparrow \downarrow)$ & 2 & $3 s^{2}$ \\
3 & 1 & $(-1,0,1)$ & $(\uparrow \downarrow, \uparrow \downarrow, \uparrow \downarrow)$ & 6 & $3 p^{6}$ \\
3 & 2 & $(-2,-1,0,1,2)$ & $(\uparrow \downarrow, \uparrow \downarrow, \uparrow \downarrow, \uparrow \downarrow, \uparrow \downarrow)$ & 10 & $3 d^{10}$ \\
4 & 3 & $(-3,-2,-1,0,1,2,3)$ & $(\uparrow \downarrow, \uparrow \downarrow, \uparrow \downarrow, \uparrow \downarrow, \uparrow \downarrow, \uparrow \downarrow, \uparrow \downarrow)$ & 14 & $4 f^{14}$ \\
4 & 3 & $(-3,-2,-1,0,1,2,3)$ & $(\uparrow, \uparrow, \uparrow, \uparrow, \uparrow, \uparrow, \uparrow)$ & 7 & $4 f^{7}$ \\
\hline
\end{tabular}

nada óbvio explicar porque o nível $4 s^{2}$ é ocupado antes do $3 d^{10}$, uma vez que possuindo maior número quântico principal o $4 s^{2}$ é mais energético e não deveria tomar a frente de um estado com $n=3$.

Curiosamente não se encontra o diagrama de Pauling em literaturas de outros países, sobretudo aquelas em inglês; é provável que a autoria pelo menos não deveria ser atribuída exclusivamente a ele. Enquanto vários autores alemães como Niels Bohr e Wolfgang Pauli contribuiram para o diagrama, o menos conhecido Erwin Madelung propôs uma regra empírica que só foi parcialmente explicada decadas depois [9]. A regra define basicamente que: "Orbitais com valores $(n+l)$ menores são ocupados primeiro". O que aprendemos como diagrama de Pauling no Brasil é equivalente a regra de Madelung.

\subsection{Spin, terras-raras e tecnologias de energia limpa}

Se na sua infância você brincou com um imã alguma vez, deve se lembrar que aqueles metais tem uma estranha propriedade de empurrar/puxar um ao outro. Isso acontece devido ao que chamamos de ferromagnetismo, que é a capacidade de um material de possuir momentos magnéticos alinhados em seu interior sem a presença de um campo externo; é como se os átomos se comportassem como pequenas bussolas que se mantém alinhadas mesmo na ausência do campo magnético terrestre. O ferromagnetismo é dependente da temperatura, uma vez que com ela se aumenta a agitação que acaba desalinhando as bussolas atômicas do material. Portanto, os diferentes imãs permanentes que podem ser encontrados/obtidos possuem diferentes intensidades de campo magnético e de resistência a temperatura.

Para comparar diferentes tipos de imãs visando sua utilização em nosso cotidiano são necessários alguns parâmetros. A magnetização residua 3 , o campo coercitivo ou coercividade $£^{4}$ e a temperatura de Curi $\S^{5}\left(T_{c}\right)$. Então, um bom imã deve produzir um campo magnético intenso, ser muito resistente a um campo externo e dependendo da aplicação pode ser necessário que sua temperatura de Curie seja alta.

Em 1967, pesquisadores da força aérea americana e da General Eletric [10] descobriram e registraram em

\footnotetext{
${ }^{3}$ Medida em Teslas, é a intensidade do campo produzido.

${ }^{4}$ Medida em Oersted, é a resistência do material a se demagnetizar.

${ }^{5}$ Medida em Celcius, acima dela o material deixa de ser ferromagnético.
}

1969 um pedido de patente de um novo e promisor tipo de imã permanente, que não era baseado nas ferrita: 6 mundialmente utilizadas desde o século anterior. A liga do novo imã era baseada em Samário e Cobalto, nos sistemas $\mathrm{SmCo}_{5}$ ou $\mathrm{Sm}_{2} \mathrm{Co}_{17}$, ainda que diversos outros materiais tenham sido investigados 10,11$]$. As vantagens do Sm-Co com relação as ferritas eram grandes: campos de 1.1 contra 0.2 Tesla; coercividade de 1600 contra 300 Oersted e $T_{c}$ de $\sim 750^{\circ} \mathrm{C}$ contra $\sim 450^{\circ} \mathrm{C}$.

Outra importante liga magnética foi desenvolvida nos anos 1980, por pesquisadores da General Motors [12] e da Sumitomo Special Metals 13, certamente motivados pela alta no preço do petróleo durante a década anterior que fomentou projetos de veículos elétricos. Eles descobriram uma liga do sistema $N d_{2} F e_{14} B$, com pequenas variações na concentração e no cozimento com as quais se pode obter um campo magnético ainda maior que no sistema Sm-Co, chegando a 1.4 Tesla; coercividade de 2000 Oersted, porém, com uma menor resistência a temperatura $\operatorname{com} T_{c} \sim 300^{\circ}$. Essa fragilidade com a temperatura pode ser parcialmente melhorada pela substituição de parte do Neodímio pelo Disprósio, o que encarece e limita a produção devido a escassez ainda maior deste elemento químico.

A explicação de porque estes imãs especiais estão intimamente ligados com os metais de terras-raras, como o Samário e o Neodímio, tem uma ligação fundamental com o spin dos elétrons. Sem ter toda essa teoria consolidada, as curiosas propriedades magnéticas dos terras-raras já eram estudadas na decada de 1930, por exemplo num interessante trabalho de Amelie Frank [14] com o Samário e o Európio.

Todos os elementos da família dos terras-raras, ou Lantanídeos, possuem seus últimos elétrons na camada $4 \mathrm{f}$. Se você notar na tabela 1 , verá que uma particularidade dessa camada é o grande número de 14 elétrons que ela comporta; mais interessante ainda é lembrar que na ordem de preenchimento das camadas vamos primeiramente ter 7 Spins paralelos e só a partir do oitavo elétron haverá Spin antiparalelos.

Isso implica que dependendo da estrutura cristalina da liga contendo terra-rara e portanto das ligações químicas que estes átomos fazem, eles podem ter até 7 elétrons com Spin paralelo num dado cristal; elementos menores, como o ferro, vão possuir um limite menor devido ao fato

\footnotetext{
${ }^{6}$ Uma cerâmica a base de óxido de ferro.
} 
de que seus elétrons ficam numa camada que comporta menos elétrons desemparelhados do que a $\mathrm{f}$.

\subsection{Contexto atual}

A iminente poluição do planeta devido ao uso intensivo de combustíveis fósseis somada a uma eventual escassez dessa fonte de energia tem fomentado as chamadas tecnologias verdes ou de baixa emissão de $\mathrm{CO}_{2}$, e em várias delas os terras-raras são fundamentalmente importantes devido a particularidade dos Spins em sua eletrosfera. Além desses fatores, nos geradores eólicos por exemplo, imãs contendo Neodímio e Disprósio são utilizados para construir aerogeradores maiores e mais potentes; os imãs são mais leves que as alternativas disponíveis, e assim fica mais barato construir toda a estrutura que mantém o aerogerador a até 150 metros de altura.

Entretanto, ao mesmo tempo que os terras-raras são a chave para diversas tecnologias, eles também são dos principais limitantes para a adoção em larga escala de produtos que demandam esses metais 15 17. O desenvolvimento de motores e geradores elétricos competitivos que dispensem os terras-raras é um grande desafio [18,19, colocando os terras-raras no centro de discussões políticas e econômicas [20]. Grandes consumidores de terras-raras como a Alemanha, Japão e Estados Unidos ficam numa posição frágil frente ao quase monopólio chinês da produção, de maneira que o tema dos terras-raras já é objeto de conflito comercial e político 21.

Há intensas pesquisas com novos materiais que, eventualmente, a partir de uma engenharia em escala nanométrica possam substituir os terras-raras em algumas aplicações. No caso dos aerogeradores de eletricidade, uma das abordagens consiste em utilizar eletro-imãs construídos com supercondutores [19]; suportando correntes muito maiores que os condutores convencionais, com estes materiais é possível obter um desempenho magnético para o gerador comparável com o obtido com os imãs de neodímio e disprósio. Entretanto, temperaturas próximas de $-260^{\circ} \mathrm{C}$ são necessárias para que os materiais tenham as propriedades supercondutoras, e os melhores materiais disponíveis precisam de outros elementos escassos e/ou caros, como a prata.

A história do momento magnético intrínseco o faz ser ainda hoje chamado de Spin. Esta propriedade que aparentemente vêm da "física teórica" é amplamente empregada pelo homem, seja no entendimento de como funciona a interação entre a matéria em escala atômica ou na construção de dispositivos de alta tecnologia: de discos rigídos para armazenamento de dados até tecnologias de energia renovável. Esta última particularmente importante no contexto atual quando consideramos por exemplo o papel estratégico do Neodímio e do Disprósio, que já são objeto de conflito político entre nações.

\section{Conclusão}

Após alguns semestres introduzindo o Spin em sala de aula da maneira apresentada na seção anterior, pude ve- rificar uma melhora do interesse dos alunos pelo assunto; esta afirmação é baseada apenas na minha percepção, não sendo um dado científico.

Essa abordagem também atinge outras dimensões do ensino, mostrando a ciência como um espaço de debate e não de conclusões arrogantes. Ela ilustra para os alunos que o assunto é delicado, e que se passaram décadas até que fosse possível ter uma teoria sólida que explicasse o Spin. O que não significa, por exemplo, que Zeeman estava errado ao pensar num elétron girante; afinal o elétron nem sequer havia sido descoberto em 1897.

Com isso a abordagem também contribui para a discussão entre as interpretações da mecânica quântica, ilustrando para os alunos que enquanto todos estes aspectos da teoria ondulatória da matéria não eram totalmente entendidos, era natural que muitos cientistas tivessem certa resistência em aceitar a teoria; e que isso é saudável e naturalmente faz parte do processo de desenvolvimento científico.

\section{Referências}

[1] M. P. Belançon. Revista Brasileira de Ensino de Física, 39, 4, (2017).

[2] G. G. Gomes and M. Pietrocola. Revista Brasileira de Ensino de Física, 33, 2, (2011).

[3] P. Zeeman. Nature, 55, 1424, (1897).

[4] S. J. Barnett. Reviews of Modern Physics, 7, 2, (1935).

[5] G. E Uhlenbeck and S Goudsmit. Nature, 117, 2938, (1926).

[6] E. Schrödinger. Physical Review, 28, 6, (1926).

[7] L. Pauling. Proc. Natl. Acad. Sci., 14, 4, (1928).

[8] P. A. M. Dirac. Proceedings of the Royal Society A: Mathematical, Physical and Engineering Sciences, 43, 601, (1927).

[9] D. P. Wong. Journal of Chemical Education, 56, 11, (1979).

[10] K. Strnat, G. Hoffer, J. Olson, W. Ostertag and J. J. Becker. Journal of Applied Physics, 38, 3, (1967).

[11] J. J. Becker. Journal of Applied Physics, 41, 3, (1970).

[12] J. J. Croat, J. F. Herbst, R. W. Lee and F. E. Pinkerton. Journal of Applied Physics, 55, 6, (1984).

[13] M. Sagawa, S. Fujimura, N. Togawa, H. Yamamoto and Y. Matsuura. Journal of Applied Physics, 55, 6, (1984).

[14] A. Frank. Physical Review, 39, (1932).

[15] S. Massari and M. Ruberti. Resources Policy, 38, 1, (2013).

[16] M. Z. Jacobson and M. A. Delucchi. Energy Policy, 39, 3, (2011)

[17] L. Grandell, A. Lehtil, M. K., T. Koljonen, S. Kihlman, and L. S. Lauri. Renewable Energy, 95, 2016.

[18] M. J. Kramer, R. W. McCallum, I. A. Anderson and S. Constantinides. The Journal of The Minerals, Metals \& Materials Society, 64, 7, (2012).

[19] J. Lloberas, A. Sumper, M. Sanmarti and X. Granados. Renewable and Sustainable Energy Reviews, 38, (2014).

[20] L. Baldi, M. Peri and D. Vandone. Energy Policy, 66, (2014).

[21] M. Humphries. Rare earth elements: the global supply chain. (DIANE Publishing, Washington D.C., 2010). 